University of South Carolina

Scholar Commons

$11-1999$

\title{
Validity of the Previous Day Physical Activity Recall (PDPAR) in Fifth-Grade Children
}

Stewart G. Trost

Dianne S. Ward

Ben McGraw

Russell R. Pate

University of South Carolina - Columbia, rpate@mailbox.sc.edu

Follow this and additional works at: https://scholarcommons.sc.edu/

sph_physical_activity_public_health_facpub

Part of the Public Health Commons

\section{Publication Info}

Published in Pediatric Exercise Science, Volume 11, Issue 4, 1999, pages 341-348.

Trost, S. G., Ward, D. S., McGraw, B., \& Pate, R. R. (1999). Validity of the Previous Day Physical Activity Recall (PDPAR) in fifth-grade children. Pediatric Exercise Science, 11(4), 341-348.

(C) Pediatric Exercise Science, 1999, Human Kinetics

This Article is brought to you by the Physical Activity and Public Health at Scholar Commons. It has been accepted for inclusion in Faculty Publications by an authorized administrator of Scholar Commons. For more information, please contact digres@mailbox.sc.edu. 


\title{
Validity of the Previous Day Physical Activity Recall (PDPAR) in Fifth-Grade Children
}

\author{
Stewart G. Trost, Dianne S. Ward, Ben McGraw, \\ and Russell R. Pate
}

\begin{abstract}
This study evaluated the validity of the Previous Day Physical Activity Recall (PDPAR) self-report instrument in quantifying after-school physical activity behavior in fifth-grade children. Thirty-eight fifth-grade students (mean age, 10.8 $\pm 0.1 ; 52.6 \%$ female; $26.3 \%$ African American) from two urban elementary schools completed the PDPAR after wearing a CSA WAM 7164 accelerometer for a day. The mean within-subject correlation between self-reported MET level and total counts for each 30-min block was 0.57 (95\% C.I., 0.51-0.62). Selfreported mean MET level during the after-school period and the number of 30min blocks with activity rated at $\geq 6$ METs were significantly correlated with the CSA outcome variables. Validity coefficients for these variables ranged from 0.35 to $0.43(p<.05)$. Correlations between the number of 30 -min blocks with activity rated at $\geq 3$ METs and the CSA variables were positive but failed to reach statistical significance $(r=0.19-0.23)$. The PDPAR provides moderately valid estimates of relative participation in vigorous activity and mean MET level in fifth-grade children. Caution should be exercised when using the PDPAR to quantify moderate physical activity in preadolescent children.
\end{abstract}

Promotion of lifelong physical activity in children and adolescents is a priority area for public health authorities $(4,6,14)$. Yet, without precise, well validated measures of physical activity, it is difficult for researchers and-or practitioners to: (a) document the frequency and distribution of physical activity in defined population groups; (b) identify the psychosocial and environmental factors that influence youth physical activity behavior; and (c) evaluate the effectiveness of programs to increase habitual physical activity in youth.

To date, a wide range of methods have been used to quantify physical activity behavior in children and adolescents. These include self-report questionnaires, direct observation, heart rate monitoring, activity monitors, and doubly labeled water (3). However, due to their low cost and ease of administration, self-report questionnaires are the most commonly used method for quantifying physical activity behavior in population-based research $(3,15)$.

S.G. Trost, B. McGraw, and R.R. Pate are with the Department of Exercise Science at the University of South Carolina, Columbia SC 29208. D.S. Ward is with the School of Public Health at the University of North Carolina at Chapel Hill, Chapel Hill, NC 27599. 
The Previous Day Physical Activity Recall (PDPAR) is a self-report instrument designed specifically for the cognitive abilities of children and adolescents. To help students recall their past behavior accurately, the previous day is segmented into 30-min time blocks that, in turn, are grouped into broader time periods such as morning, lunchtime, afternoon, and evening. To further enhance the quality of the data recorded, the instrument provides a numbered list of commonly performed activities grouped into the following broad categories: eating, sleeping/bathing, transportation, work/school, spare time, play/recreation, and exercise/workout.

To date, only one study has assessed the psychometric properties of the PDPAR. Weston, Petosa, and Pate (25) evaluated the reliability and validity of the PDPAR in a sample of predominantly white junior and senior high school students. The correlation between relative energy expenditure as estimated by the PDPAR and pedometer and Caltrac accelerometer counts was 0.88 and 0.77 , respectively, indicating a high degree of instrument validity. The test-retest reliability coefficient for the PDPAR administered twice within one hour was an impressive 0.98 .

The short recall period and design features of the PDPAR make it a suitable candidate for use among elementary school children. However, to date, the validity of the PDPAR instrument has not been evaluated in this population. Therefore, the purpose of this study was to evaluate the validity of the PDPAR in quantifying after-school physical activity in a diverse sample of fifth-grade children.

\section{Methods}

\section{Subjects}

Subjects for this study were 38 fifth-grade students (20 females, 18 males) from two elementary schools in Columbia, South Carolina. Thirteen of the 38 students (26.3\%) were African American. The mean age was $10.8 \pm 0.1$ years. Prior to participation in the study, written informed consent was obtained from each participant and his or her primary guardian. The study was approved by the University of South Carolina Institutional Review Board.

\section{Self-Report Instrument}

A full description of the PDPAR instrument and its scoring protocol can be found elsewhere (25). Briefly, the after-school version of the PDPAR makes use of a standardized form organized into seventeen 30-min blocks, beginning at 3:00 P.M. and continuing through 11:30 P.M. Thirty-five common activities, including sedentary activities such as television watching, are listed on the form, and each student enters the main activity in which he or she participated during each of the 30min periods. For each block, the student rates the intensity of the designated activity as very light, light, moderate, or hard. To help students select the correct level of intensity, the instrument includes cartoon illustrations depicting activities representative of each intensity level.

\section{Criterion Physical Activity Measure}

Objective assessments of physical activity behavior were obtained using the Computer Science and Applications Inc. (CSA) 7164 activity monitor (Shalimar, FL). 
The CSA 7164 is a uniaxial accelerometer designed to detect vertical acceleration ranging in magnitude from 0.05 to $2.00 \mathrm{Gs}$ with frequency response of 0.25 to 2.50 $\mathrm{Hz}$. These parameters allow for the detection of normal human motion and will reject high frequency vibrations encountered in activities such as operation of a lawn mower. The filtered acceleration signal is digitized, and the magnitude is summed over a userspecified time interval. At the end of each interval, the summed value or activity "count" is stored in memory, and the integrator is reset. For the present study, a 1-min sampling interval was used. The CSA 7164 has been shown to be a valid and reliable tool for assessing physical activity in children aged 10 to 14 years (23).

\section{Protocol}

The study was conducted over 2 consecutive days during the spring of 1996 . Before the start of school on Day 1, participating students were outfitted with a single CSA 7164 activity monitor. Consistent with previous studies, activity monitors were attached to an adjustable elastic belt and worn over the right hip. After receiving detailed instructions regarding the care and use of the monitors, students were instructed to wear the CSA monitor until bedtime. On the morning of the second day, the monitors were collected, and stored activity counts were downloaded to a personal computer for subsequent analysis. Following removal, students completed the PDPAR for the after-school period of the previous day. All PDPAR assessments were performed in the classroom under the supervision of two trained research assistants.

\section{Data Reduction}

PDPAR. Based on the activity and the level of intensity selected, each 30-min block was assigned a literature-based metabolic equivalent task value (MET). MET values were then averaged to derive an estimate of mean relative energy expenditure during the after-school period. In addition, the number of 30-min blocks in which relative energy expenditure was 3 METs or greater and 6 METs or greater were counted to provide an index of participation in moderate-to-vigorous physical activity and vigorous physical activity, respectively. (Note: 1 MET represents the energy expenditure associated with quiet sitting $\sim 1 \mathrm{kcal} / \mathrm{kg} / \mathrm{hr}$ or $3.5 \mathrm{ml} \mathrm{O}_{2} / \mathrm{kg} / \mathrm{min}$ ).

For activity numbers that required a write-in response (jobs, hobbies, and other), a MET value based on the reported intensity of the activity was obtained from "The Compendium of Physical Activities" (1). In situations where the combination of an activity type and intensity level was considered incompatible (e.g., the activity of meal and the intensity rating of hard), the block in question was assigned a MET value considered appropriate for the given activity. If a student made four or more incompatible responses, it was assumed that he or she did not understand the rating scale, and the recall was excluded from the analyses.

CSA Activity Monitor. Stored minute-by-minute activity counts were uploaded to a software program written by the primary author for the determination of total activity counts and time spent in moderate-to-vigorous physical activity ( $\geq 3$ METs) (MVPA) during each 30-min time period of the monitoring day. Counts were converted to units of relative energy expenditure using the regression equation developed by Freedson and colleagues (10). Total activity counts and minutes of MVPA for the entire after-school period were calculated by summing the scores from each of the 30-min time blocks between 3:00 P.M. and 11:30 P.M. 


\section{Statistics}

Relationships between the CSA and PDPAR outcome variables were assessed using Spearman rank order correlation coefficients. Spearman correlation coefficients were used instead of Pearson product-moment correlation coefficients because of the skewed distributions of both sets of physical activity variables. In addition, for each subject, we calculated the correlation between self-reported MET level and total CSA counts from each 30-min time block of the after-school period. The mean within-subject correlation and its $95 \%$ confidence interval was then calculated using the Fisher-z transformation method. Significance was set at
an alpha level of 0.05 .

\section{Results}

Descriptive data for the PDPAR and CSA physical activity variables are shown in Table 1. On average, males reported/exhibited higher levels of physical activity than girls; however, only the difference with respect to vigorous physical activity blocks ( $\geq 6$ METs), as measured by the PDPAR, was statistically significant at the .05 level. Self-reported estimates of vigorous and moderate-to-vigorous physical activity were similar to previous studies using the PDPAR in preadolescent youth (13, $21,24)$. Only one recall was excluded because of four or more incompatible responses.

Between-subject correlations between the PDPAR and the CSA outcome variables are shown in Table 2. Mean MET level during the after-school period and the number of 30-min blocks with activity rated at $\geq 6 \mathrm{METs}$ were significantly correlated with the CSA outcome variables. Validity coefficients for these two variables ranged from 0.35 to $0.43(p<.05)$. Correlations between the number of 30 -min blocks with activity rated at $\geq 3 \mathrm{METs}$ and the CSA variables were positive but failed to reach statistical significance $(r=0.19-0.23)$. The mean within-subject correlation between self-reported MET level and total CSA counts for each 30-min block was 0.57 . The $95 \%$ confidence interval of $0.51-0.62$ indicated that this association was statistically significant at the .05 level (Table 2 ). Thus, not only were students somewhat able to recall the mode and intensity of the previous day's activities, they were relatively successful in recalling the specific 30 -min time period during which they
engaged in activity.

Table 1 Descriptive Data for the PDPAR and CSA Physical Activity Variables

\begin{tabular}{|c|c|c|c|c|c|c|}
\hline \multirow[b]{2}{*}{ Variable } & \multicolumn{2}{|c|}{ Group $(N=37)$} & \multicolumn{2}{|c|}{ Males $(N=18)$} & \multicolumn{2}{|c|}{ Females $(N=19)$} \\
\hline & $M$ & $S D$ & $M$ & $S D$ & M & $S D$ \\
\hline Mean METs & 2.8 & 1.3 & 3.2 & 1.2 & 2.5 & 1.3 \\
\hline $\begin{array}{l}\text { \# 30-min blocks } \\
\geq 3 \text { METs }\end{array}$ & 4.8 & 3.3 & 5.8 & 3.5 & 3.9 & 3.0 \\
\hline $\begin{array}{l}\text { \# 30-min blocks } \\
\geq 6 \text { METs }\end{array}$ & 2.9 & 3.0 & 4.0 & 2.9 & 1.8 & 2.8 \\
\hline $\begin{array}{l}\text { Total CSA counts } \\
\text { CSA MVPA (min) }\end{array}$ & $\begin{array}{c}318085 \\
43.1\end{array}$ & $\begin{array}{c}197004 \\
31.6\end{array}$ & $\begin{array}{c}355550 \\
48.3\end{array}$ & $\begin{array}{c}240732 \\
32.5\end{array}$ & $\begin{array}{c}282592 \\
38.3\end{array}$ & $\begin{array}{r}141974 \\
30.8\end{array}$ \\
\hline
\end{tabular}


Table 2 Spearman Correlation Coefficients Between the PDPAR and CSA Physical Activity Variables $(N=37)$

CSA variables

PDPAR variables

Total CSA counts

CSA MVPA (min)

Mean METs

\# 30-min blocks $\geq 3$ METs

\# 30-min blocks $\geq 6$ METs

Average within-subject correlation

between self-reported MET

level and total

CSA counts for each 30-min time block
$0.39 *$

$0.43 *$

0.23

0.19

$0.35 *$

0.38 *

0.57 *

$* p<.05$.

\section{Discussion}

The PDPAR has been used in numerous studies involving preadolescent youth $(8$, $13,21,22,24)$. However, to date, no study has evaluated the validity of this instrument in children aged 11 years and under. The present study evaluated the validity of the PDPAR in an ethnically diverse sample of fifth-grade students. Our results indicate that the PDPAR is a moderately valid instrument for assessing average MET level and relative participation in vigorous physical activity in this population. However, the PDPAR does not appear to provide useful estimates of moderate physical activity in fifth-grade children.

In absolute terms, the validity coefficients for mean MET level and vigorous physical activity were low. However, when compared to previous studies using accelerometers to validate self-reports in children $(12,16-20)$, it appears that our results are typical and supportive of our conclusion that the PDPAR provides limited but useful estimates of relative participation in physical activity. Sallis et al. (19) utilized a single axis accelerometer to evaluate the validity of the Self Administered Physical Activity Checklist (SAPAC) in 119 children (mean age, $10.9 \pm 0.5$ ). The correlation between Caltrac counts and self-reported MVPA ranged from 0.22 to 0.43 . In a study of 35 obese children between the ages of 8 and 12, Coleman and colleagues (5) reported a mean correlation of $0.36 \pm 0.35$ between self-reported MET level and METs derived from triaxial accelerometry. Simons-Morton et al. (20) utilized the single axis accelerometer to validate their Physical Activity Interview (PAI). The correlation between Caltrac counts and PAI-reported minutes of MVPA was 0.63 and 0.47 for fifthand third-grade students, respectively. The slightly higher validity coefficients reported in that study are likely attributable to the PAI's structured interview format and the use of activity records to aid recall.

Objective measures such as accelerometers are frequently employed as criterion measures of physical activity in validation studies $(9,15)$. However, it is important to note that these devices do not provide error-free estimates of physical activity. Accelerometers do not accurately assess energy expenditure during non-weight 
bearing activities such as bicycling and may not be sensitive to many of the complex movement patterns exhibited by children during free play $(9,23)$. Thus, while our results underscore the need to exercise caution when using self-reports in children, it is likely that the our correlation coefficients are underestimates of the true association between the PDPAR and CSA variables (18).

The magnitude of our correlations were substantially weaker than those reported by Weston et al. (25) for junior and senior high school students. This finding is in agreement with previous validation studies in which validity coefficients for self-reported physical activity were found to be higher among adolescent youth than young children $(2,17)$. Also of concern was the finding that the number of 30 min blocks with activity $\geq 3$ METs, an index of participation in both moderate and vigorous physical activity, was only weakly correlated with the CSA variables. This observation is consistent with the conclusion that individuals have difficulty recalling moderate versus vigorous physical activity $(7,11)$. Alternatively, the low correlations may have been, in part, a reflection of the activity monitor's inability to measure commonly performed moderate intensity activities such as bicycling and unstructured play and games. In any case, caution should be exercised when using the PDPAR to quantify moderate physical activity in preadolescent children.

In addition to the problems associated with using a single axis accelerometer device as a criterion measure of physical activity, the present study had several other limitations that warrant consideration. First, logistic constraints precluded us from administering the PDPAR on multiple days. When administering the PDPAR to students, it is recommended that participants be allowed to complete a "practice PDPAR" in order to become more accustomed to the assessment protocol. Second, we cannot rule out the possibility that wearing the activity monitors on the day prior to the recall may have positively biased the results by increasing subjects' attentions to their daily activities. Lastly, our relatively small sample size precluded us from exploring potentially important gender and racial differences in validity coefficients.

In conclusion, the PDPAR is a moderately valid self-report instrument for quantifying relative participation in vigorous physical activity and mean MET level in fifth-grade children. However, substantial caution should be exercised when utilizing the PDPAR to quantify moderate intensity physical activity in this population. Future studies should evaluate the validity of the PDPAR in elementary school children using a variety of objective methods. Direct observation or a combination of three-dimensional accelerometers and heart rate monitors may be particularly effective. Additionally, future studies should assess physical activity over multiple days and have sufficient sample sizes to examine the validity the PDPAR in population subgroups defined by age, gender, and race/ethnicity.

\section{References}

1. Ainsworth, B.E., W.L. Haskell, A.S. Leon, D.R. Jacobs Jr., H.J. Montoye, J.F. Sallis, and R.S. Paffenbarger Jr. Compendium of physical activities: Classifications of energy costs of human physical activities. Med. Sci. Sports Exerc. 25:71-80, 1993.

2. Baranowski, T. Validity and reliability of self-report measures of physical activity: An information-processing perspective. Res. Q. Exerc. Sports. 59:314-327, 1988.

3. Baranowski, T., C. Bouchard, O. Bar-Or, T. Bricker, G. Health, S.Y.S. Kimm, R. Malina, E. Obarzanek, R.R. Pate, W.B. Strong, B. Truman, and R. Washington. Assessment, 
prevalence, and cardiovascular health benefits of physical activity and fitness in youth. Med. Sci. Sports Exerc. 24(Suppl.):S237-S247, 1992.

4. Center for Disease Control and Prevention. Guidelines for school and community programs to promote lifelong physical activity among young people. MMWR. 46:(No. RR-6), 1997.

5. Coleman, K.J., B.E. Saelens, M.D. Wiedrich-Smith, J.D. Finn, and L.H. Epstein. Relationships between TriTrac-R3D vectors, heart rate, and self-report in obese children. Med. Sci. Sports Exerc. 29:1535-1542, 1997.

6. Department of Health and Human Services. Physical Activity and Health: A Report of the Surgeon General. Atlanta, GA: U.S. Department of Health and Human Services, Centers for Disease Control and Prevention, National Center for Chronic Disease Prevention and Health Promotion, 1996.

7. Durante, R., and B.E. Ainsworth. The recall of physical activity: Using a cognitive model of the question-answering process. Med. Sci. Sports Exerc. 28:1282-1291, 1996.

8. Felton, G.M., M.A. Parsons, R.R. Pate, D.S. Ward, R. Saunders, R. Valois, M. Dowda, and S.G. Trost. Predictors of alcohol use among rural adolescents. J. Rural Health. 12:378-385, 1996.

9. Freedson, P.S. Electronic motion sensors and heart rate measures of physical activity in children. J. Sch. Health. 61:220-223, 1991.

10. Freedson, P.S., J. Sirard, E. Debold, R.R. Pate, M. Dowda, S.G. Trost, and J.F. Sallis. Calibration of the Computer Science and Applications Inc. (CSA) accelerometer. Med. Sci. Sports Exerc: 29(Suppl.):S45, 1997.

11. Jacobs, D.R., Jr., B.E. Ainsworth, T.J. Hartman, and A.S. Leon. A simultaneous evaluation of 10 commonly used physical activity questionnaires. Med. Sci. Sports Exerc. 25:81-91, 1993.

12. Janz, K.F., J. Witt, and L.T. Mohoney. The stability of children's physical activity as measured by accelerometry and self-report. Med. Sci. Sports Exerc. 27:1326-1332, 1995.

13. Pate, R.R., S.G. Trost, G.M. Felton, D.S. Ward, M. Dowda, and R. Saunders. Correlates of physical activity behavior in rural youth. Res. Q. Exerc. Sports. 68:241-248, 1997.

14. Public Health Service. Healthy People 2000: National Health Promotion and Disease Prevention Objectives (DHHS Publication No. PHS 91-50212). Washington, DC: U.S. Department of Health and Human Services, 1990.

15. Sallis, J.F. Self-report measures of children's physical activity. J. Sch. Health. 61:215219, 1991.

16. Sallis J.F., M.J. Buono, J.J. Roby, D. Carlson, and J.A. Nelson. The Caltrac accelerometer as a physical activity monitor for school-age children. Med. Sci. Sports Exerc. 22:698-703, 1990.

17. Sallis, J.F., M.J. Buono, J.J. Roby, F.G. Micale, and J.A. Nelson. Seven-day recall and other physical activity self-reports in children and adolescents. Med. Sci. Sports Exerc. 25:99-108, 1993.

18. Sallis, J.F., S.A. Condon, K.J. Goggin, J.J. Roby, B. Kolody, and J.E. Alcarez. The development of self-administered physical activity surveys for 4th grade students. Res. Q. Exerc. Sport. 64:25-31, 1993.

19. Sallis, J.F., P.K. Strikmiller, D.W. Harsha, H.A. Feldman, S. Ehlinger, E.J. Stone, J. Williston, and S. Woods. Validation of interviewer- and self-administered physical activity checklists for fifth-grade students. Med. Sci. Sports Exerc. 28:840-851, 1996.

20. Simons-Morton, B.G., W.C. Taylor, I.W. Huang. Validity of the physical activity interview and Caltrac with preadolescent children. Res. Q. Exerc. Sport. 65:84-89, 1994. 
21. Trost, S.G., R.R. Pate, M. Dowda, R. Saunders, D.S. Ward, and G. Felton. Gender differences in physical activity and determinants of physical activity in rural fifth-grade children. J. Sch. Health 66:145-150, 1996.

22. Trost, S.G., R.R. Pate, R. Saunders, D.S. Ward, M. Dowda, and G. Felton. A prospective study of the determinants of physical activity behavior in rural fifth-grade children. Prev. Med. 27:257-63, 1997.

23. Trost, S.G., D.S. Ward, S.M. Moorehead, P.D. Watson, W. Riner, and J.R. Burke. Validity of the computer science and applications (CSA) activity monitor in children. Med. Sci. Sports Exerc. 30:629-633, 1998.

24. Ward, D.S., S.G. Trost, G. Felton, R. Saunders, M.A. Parson, M. Dowda, and R.R. Pate. Physical activity and physical fitness in African-American girls with and without obesity. Obesity Res. 5:572-577, 1997.

25. Weston, A.T., R. Petosa, and R.R. Pate. Validation of an instrument for measurement of physical activity in youth. Med. Sci. Sports Exerc. 29:138-143, 1997.

\section{Acknowledgment}

Funding for this study was provided by the Centers for Disease Control and Prevention, grant number U48/CCU409664. 